\title{
IN-VITRO INHIBITION OF MYCOBACTERIA BY VIRIDANS STREPTOCOCCI
}

\author{
B. W. Allen \\ Medical Research Council Unit for Laboratory Studies of Tuberculosis, Royal \\ Postgraduate Medical School, Ducane Road, London W12 OHS
}

\begin{abstract}
SUmmaRY. Eleven strains of Mycobacterium tuberculosis and 13 other species of mycobacteria were examined for susceptibility to 26 strains of viridans streptococci. Susceptibility was detected by the simultaneous antagonism technique, but not by tests of deferred antagonism. It is concluded that in-vitro inhibition of mycobacterial growth by actively growing streptococci is due to a peroxide-mediated antagonism which is too variable to be of value for identification. However, a standard bactericidal test with hydrogen peroxide is worth investigation because this might provide a simple inexpensive aid for the identification of $M$. avium-intracellulare serotypes.
\end{abstract}

\section{INTRODUCTION}

In a previous report (Allen and Swaffield, 1982) Streptococcus sanguis II and $S$. mitis were shown to be the main cause of delayed contamination of cultures of Mycobacterium tuberculosis. On further examination of a few strains of streptococci, in-vitro antagonism towards $M$. tuberculosis was observed. Other workers have reported streptococcal antagonism to mycobacteria. Gelbert, Tonaki and Adams (1974) isolated, but did not identify, an $\alpha$-haemolytic streptococcus that inhibited growth of $M$. intracellulare, $M$. avium and other closely related species. Darling and Hart (1976) described the antagonistic action of $S$. salivarius and $S$. faecalis to $M$. tuberculosis, $M$. kansasi and scotochromogenic species, but the two strains they tested did not inhibit growth of $M$. intracellulare, $M$. avium or $M$. fortuitum. It has been suggested (Gelbert et al., 1974; Darling and Hart, 1976) that this apparently selective antagonism might be of value for the in-vitro identification or typing of mycobacteria. The present investigation was designed to identify the nature of streptococcal antagonism and to determine its value in the identification of mycobacteria.

\section{MATERIALS AND METHODS}

Bacterial strains. The "producer" strains of streptococci were from a collection of cultures isolated from contaminated Lowenstein-Jensen $(\mathrm{LJ})$ cultures during a previous investigation (Allen and Swaffield, 1982). The 26 strains used were $S$. sanguis II (17 strains) and S. mitis (9). Purity checks were made on blood-agar medium and cultures were stored as a suspension at $-196^{\circ} \mathrm{C}$. Cultures used as indicators of antagonism were 11 strains of $M$. tuberculosis and 13 
TABLE I

Indicator strains of $M$. tuberculosis

\begin{tabular}{llccc}
\hline Strain no. & Origin & $\begin{array}{c}\text { Virulence for } \\
\text { guinea-pig }\end{array}$ & Phage type & $\begin{array}{c}\text { Susceptibility to } \\
\text { peroxide* }\end{array}$ \\
\hline I2646 & Britain & High & A & R \\
79499 & Madras & High & A & R \\
J6729 & Burma & High & A & R \\
79112 & Burma & Low & I & S \\
79157 & Madras & Low & I & S \\
79500 & Madras & Low & I & R \\
J6720 & Teheran & High & B & R \\
J6198 & Britain & High & B & R \\
J6839 & Burma & High & B & R \\
H37Rv & USA & High & B & Not tested \\
B1453† & Britain & Low & S \\
\hline
\end{tabular}

* Percentage of cfu surviving exposure to $0.02 \% \mathrm{H}_{2} \mathrm{O}_{2}$ for 90 min at $37^{\circ} \mathrm{C}$. Resistant $(\mathrm{R})>10 \%$ survivors; sensitive $(\mathrm{S}) \approx 10 \%$ survivors.

$\dagger$ Isoniazid-resistant (MIC $>50 \mathrm{mg} / \mathrm{L}$ in $\mathrm{LJ}$ medium) catalase-negative strain.

TABLE II

Indicator strains of mycobacteria other than M. tuberculosis

\begin{tabular}{ll}
\hline Species & \multicolumn{1}{c}{ Origin } \\
\hline M. africanum & Pasteur Institute Paris \\
$M$. avium & NCTC8559 \\
M. bovis & Human isolate \\
M. bovis var BCG & Glaxo strain \\
M. flavescens & NCTC10271 \\
M. fortuitum & NCTC10394 \\
M. gordonae & NCTC10267 \\
M. intracellulare & NCTC10425 \\
M. kansasi & NCTC10268 \\
$M$. marinum & NCTC2275 \\
$M$. phlei & NCTC10266 \\
$M$. scrofulaceum & NCTC10803 \\
$M$. smegmatis & ATCC607 \\
\hline
\end{tabular}

NCTC: National Collection of Type Cultures, Central Public Health Laboratory, Colindale Avenue, London NW9 5HT.

strains of other species of mycobacteria. Strains of $M$. tuberculosis with well defined in-vitro and in-vivo characteristics were selected from the culture collection in this department. The main characteristics of these strains are presented in table I. Table II lists the origin of the other strains that were selected to provide a representative sample of species of mycobacteria. All cultures of mycobacteria were stored as a suspension at $-196^{\circ} \mathrm{C}$.

Culture media. Liquid cultures of streptococci were grown in Todd-Hewitt Broth (THB) (Difco 0492) for $24 \mathrm{~h}$ at $37^{\circ} \mathrm{C}$. Liquid cultures of mycobacteria were grown in $7 \mathrm{H} 9$ Tween-albumin Medium (Difco 0713) at $37^{\circ} \mathrm{C}$. Slow-growing mycobacteria were incubated for 14 days and rapid growing species for 4 days.

Solid media for the detection of antagonism were Oleic Acid Albumin Agar (7H11) (Difco 0838) and LJ medium. Plates of 7H11 agar, supplemented with Oleic acid-albumin-dextrosecatalase Complex (Difco 0722), were prepared by pouring $20 \mathrm{ml}$ of molten agar into $9-\mathrm{cm}$ diameter plastic or glass petri dishes. Slopes of LJ medium, with or without benzylpenicillin 60 $\mathrm{mg} / \mathrm{L}$, were prepared in $25-\mathrm{ml}$ screw-capped glass containers and solidified by inspissation at $85^{\circ} \mathrm{C}$ for $60 \mathrm{~min}$. 
Simultaneous antagonism. Four wells $8.0 \mathrm{~mm}$ in diameter were cut in $7 \mathrm{H} 11$-agar plates by means of a sterile metal punch. Plates were inoculated by spreading $0 \cdot 1 \mathrm{ml}$ of indicator culture over the agar surface with a glass rod. Four drops (about $0.12 \mathrm{ml}$ ) of producer cultures in THB were delivered into each well. Plates were sealed in polyethylene bags and incubated at $37^{\circ} \mathrm{C}$ for 5 days (rapid growers) or 21 days (slow growers). The resultant zones of inhibition were measured with vernier callipers. Tests were also made on $\mathrm{LJ}$ medium, with and without benzylpenicillin, with $M$. tuberculosis strains 12646 and B1453 and $M$. intracellulare as indicators. Slopes of media were inoculated with $10 \mu \mathrm{l}$ of a tween-albumin culture of the mycobacteria. Paper disks, $5 \mathrm{~mm}$ in diameter and soaked in a THB culture of streptococci, were placed on the centre of each slope. Cultures were incubated for 3 weeks at $37^{\circ} \mathrm{C}$.

Deferred antagonism. Producer strains were inoculated as a streak, approximately $10 \mathrm{~mm}$ wide, on duplicate $7 \mathrm{H} 11$ agar plates in glass petri dishes. One culture from each pair was incubated at $37^{\circ} \mathrm{C}$ for $48 \mathrm{~h}$ and the other culture at $37^{\circ} \mathrm{C}$ for 14 days. After incubation, growth was removed with a glass microscope slide and the plate exposed to chloroform vapour for 30 $\mathrm{min}$. To remove residual chloroform, agar surfaces were exposed to a laminar airflow for $30 \mathrm{~min}$. Indicator strains were inoculated at right angles to the producer streak culture with a metal applicator which delivered nine indicator cultures simultaneously. The nine indicator strains were $M$. tuberculosis strains I2646, 79112, J6720, H37Rv and B1453, M. kansasi, $M$. intracellulare, $M$. bovis and $M$. bovis var BCG. Plates were incubated until macroscopic growth could be recorded for all strains.

Inhibitory action of culture fractions. Cultures of streptococci in THB, cell-free filtrates, and washed cells were examined for inhibitory effect against $M$. tuberculosis strains I2646 and B1453, $M$. kansasi and $M$. intracellulare by the simultaneous antagonism technique. Overnight THB cultures $(10 \mathrm{ml})$ were used to test complete cultures. Cell-free filtrates were prepared by centrifuging cultures and filtering the supernatant fluid through $0 \cdot 22-\mu \mathrm{m}$ pore-size Sterive ${ }^{\circledR} \mathrm{GS}$ filter units (Millipore Corporation). Deposited cells were washed three times in 10-ml volumes of Hanks's Buffered Salt Solution (HBSS) (Gibco Europe 4025) then resuspended in $10 \mathrm{ml}$ of HBSS.

Inactivation of inhibitory effect by catalase was studied by the following techniques: plates of $7 \mathrm{H} 11$ agar containing purified thymol-free catalase powder from bovine liver at concentrations of 10,100 and 1000 Sigma units/ml were used to detect antagonism by streptococci against $M$. tuberculosis (strains I2646 and B1453) and $M$. intracellulare by the simultaneous antagonism technique. The effect of catalase diffusion was studied on $7 \mathrm{H} 11$ agar plates in which two ditches, approximately $5 \mathrm{~mm} \times 70 \mathrm{~mm}$, were made with a metal cutter. The ditches were separated by about $8 \mathrm{~mm}$ at the closest point and about $40 \mathrm{~mm}$ at the most distant point, thus forming an unconnected " $V$ " shape. Plates were surface inoculated with $M$. tuberculosis strains I2646 and B1453 and $M$. intracellulare. One ditch was filled with catalase solution 1000 Sigma units $/ \mathrm{ml}$ and the other with overnight THB culture of streptococci. By these techniques the three strains of mycobacteria were tested against 26 cultures of streptococci. Plates were incubated as in previous experiments.

The $p H$ within zones of inhibition produced by streptococci on plates seeded with $M$. tuberculosis strain B1453, $M$. kansasi, $M$. intracellulare and $M$. bovis var BCG was measured with a Radiometer PHM63 digital $p \mathrm{H}$ meter fitted with a Russel 3-mm stem spear combination electrode.

Detection of peroxide production during growth of streptococci was by the benzidine blood agar (BBA) method (Kraus et al., 1957). Heart Infusion Agar (Difco 044) with lysed horse red

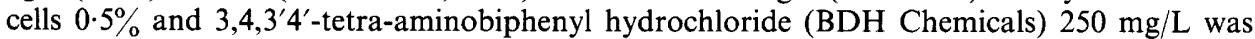
poured in $20-\mathrm{ml}$ volumes in 9-cm petri dishes. Plates were surface inoculated with cultures of streptococci and incubated aerobically at $37^{\circ} \mathrm{C}$ for 4 days. Duplicate tests were incubated in anaerobic conditions.

Susceptibility to hydrogen peroxide. M. tuberculosis strains H37Rv and B1453, M. bovis var BCG and $M$. intracellulare were tested for peroxide susceptibility by the method of Subbaiah $e t$ al. (1960). In brief, $0.2 \mathrm{ml}$ of each culture, grown in $7 \mathrm{H} 9$ medium, was incubated with $3.8 \mathrm{ml}$ of phosphate buffer at $p \mathrm{H} 7.0$ for $90 \mathrm{~min}$ (buffer control), and an additional $0.2 \mathrm{ml}$ was incubated with $3.8 \mathrm{ml}$ of buffer containing $0.02 \% \mathrm{v} / \mathrm{v} \mathrm{H}_{2} \mathrm{O}_{2}$ (test) for $90 \mathrm{~min}$ at $37^{\circ} \mathrm{C}$. After incubation, viable counts were set up on samples from the buffer control and the test. The percentage of 
organisms surviving exposure was calculated by dividing the number of colony-forming units (cfu) in the suspension exposed to $\mathrm{H}_{2} \mathrm{O}_{2}$ by the number of cfu in the buffer control, and multiplying by 100 .

\section{RESULTS}

An example of streptococci inhibiting $M$. tuberculosis, by the simultaneous antagonism test, is illustrated in fig. 1. Zones of inhibition ranged from $13 \mathrm{~mm}$ to 40 $\mathrm{mm}$, but the characteristic rough colonial morphology of $M$. tuberculosis often made accurate measurement difficult. As shown in table III, there was no association between inhibition by streptococci and the well defined characteristics of 10 of the 11 strains of $M$. tuberculosis used as indicators. However, strain B1453, an isoniazid-resistant catalase-negative strain, was susceptible to $25(96 \%)$ strains of streptococci. Table IV lists the results obtained when 13 other species of mycobacteria were cultured simultaneously with streptococci. $M$. africanum, $M$. avium, $M$. bovis, $M$. fortuitum, $M$. gordonae, $M$. phlei and $M$. scrofulaceum were resistant to most strains of streptococci. However, $M$. intracellulare was inhibited by all 26 strains of streptococci and $M$. bovis

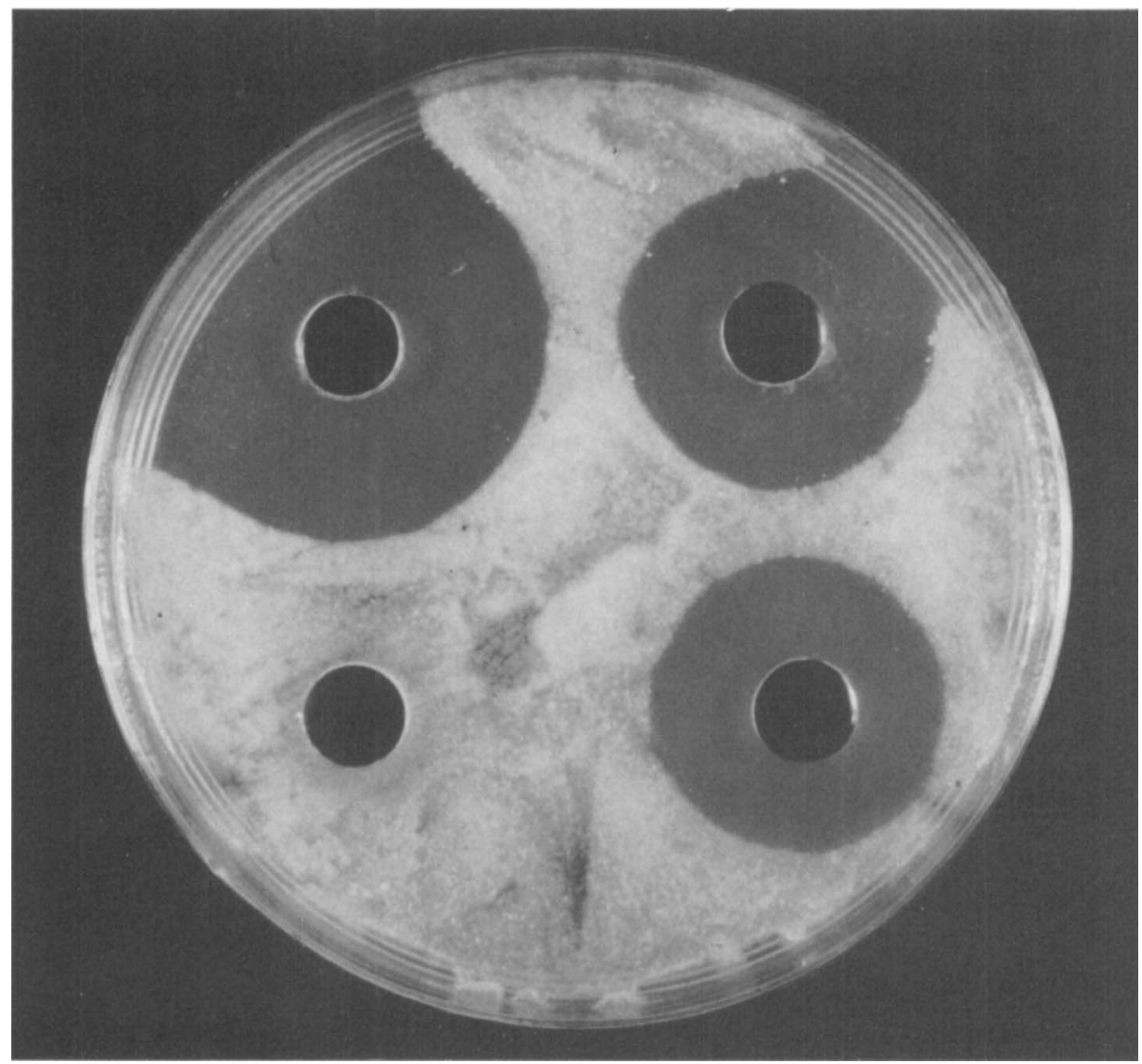

FIG. 1.-An example of variable streptococcal inhibition of $M$. tuberculosis on oleic-acid albumin agar. 
TABLE III

Effect of streptococci on strains of M. tuberculosis by the simultaneous antagonism technique

\begin{tabular}{lc}
$\begin{array}{l}\text { Strain of } \\
M . \text { tuberculosis }\end{array}$ & $\begin{array}{c}\text { Number (and percentage) of 26 strains } \\
\text { of streptococci producing } \\
\text { zones of inhibition }\end{array}$ \\
\hline $\mathrm{I} 2646$ & $13(50)$ \\
79499 & $5(19)$ \\
$\mathrm{J} 6729$ & $1(4)$ \\
79112 & $6(23)$ \\
79157 & $1(4)$ \\
79500 & $14(54)$ \\
$\mathrm{J} 6720$ & 0 \\
$\mathrm{~J} 6198$ & $4(15)$ \\
$\mathrm{J} 6839$ & $2(8)$ \\
H37Rv & $6(23)$ \\
B1453 & $25(96)$ \\
\hline
\end{tabular}

TABLE IV

Inhibitory effect of streptococci on different species of mycobacteria

\begin{tabular}{lc}
\hline Species & $\begin{array}{c}\text { Number (and percentage) of 26 strains } \\
\text { of streptococci producing } \\
\text { zones of inhibition }\end{array}$ \\
\hline M. africanum & $1(4)$ \\
M. avium & 0 \\
M. bovis & $2(8)$ \\
M. bovis var BCG & $23(88)$ \\
M. flavescens & $6(23)$ \\
M. fortuitum & 0 \\
M. gordonae & $1(4)$ \\
M. intracellulare & $26(100)$ \\
M. kansasi & $9(35)$ \\
M. marinum & $5(19)$ \\
M. scrofulaceum & $2(8)$ \\
M. smegmatis & $1(4)$ \\
\hline
\end{tabular}

TABLE V

Effect of streptococcal culture fractions on four cultures of mycobacteria

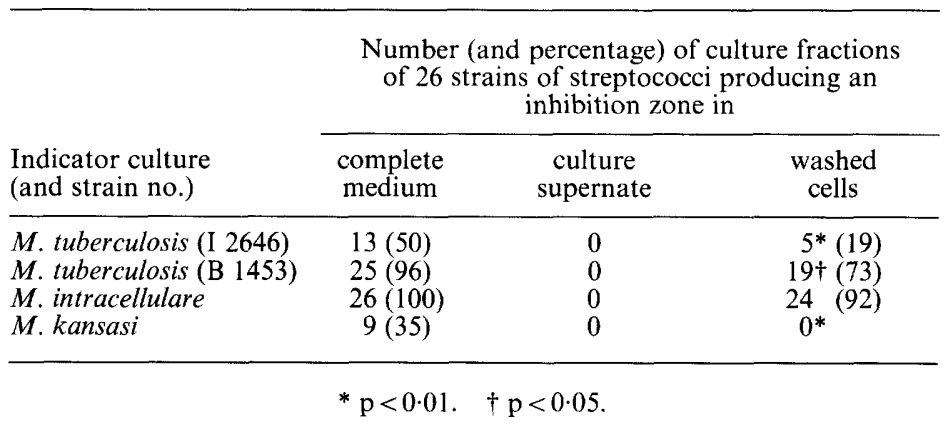


TABLE VI

Effect of catalase on streptococcal inhibition of mycobacteria during simultaneous growth on oleic-acid albumin agar

\begin{tabular}{lccc}
\hline & \multicolumn{2}{c}{$\begin{array}{c}\text { Number (and percentage) of 26 strains of streptococci } \\
\text { producing an inhibition zone in the presence of } \\
\text { catalase at a concentration (Sigma units/ml) of }\end{array}$} \\
\cline { 2 - 4 } $\begin{array}{l}\text { Indicator culture } \\
\text { (and strain no.) }\end{array}$ & 10 & 100 & 1000 \\
\hline $\begin{array}{l}\text { M. tuberculosis (I2646) } \\
\text { M. tuberculosis (B1453) }\end{array}$ & $13(50)$ & $1(4)^{*}$ & 0 \\
M. intracellulare & $25(96)$ & $22(85)$ & $1(4)^{*}$ \\
\hline
\end{tabular}

${ }^{*} \mathrm{p}=<0.001$.

var BCG by $23(88 \%)$. In similar tests on $\mathrm{LJ}$ medium, zones of inhibition occurred on drug-free medium but not on medium containing benzylpenicillin. Although the THB inoculum used on paper disks was considerably less $(0.01 \mathrm{ml})$ than that used in wells on $7 \mathrm{H} 11$ agar plates $(0 \cdot 12 \mathrm{ml}), 5(19 \%)$ strains of streptococci were antagonistic to $M$.

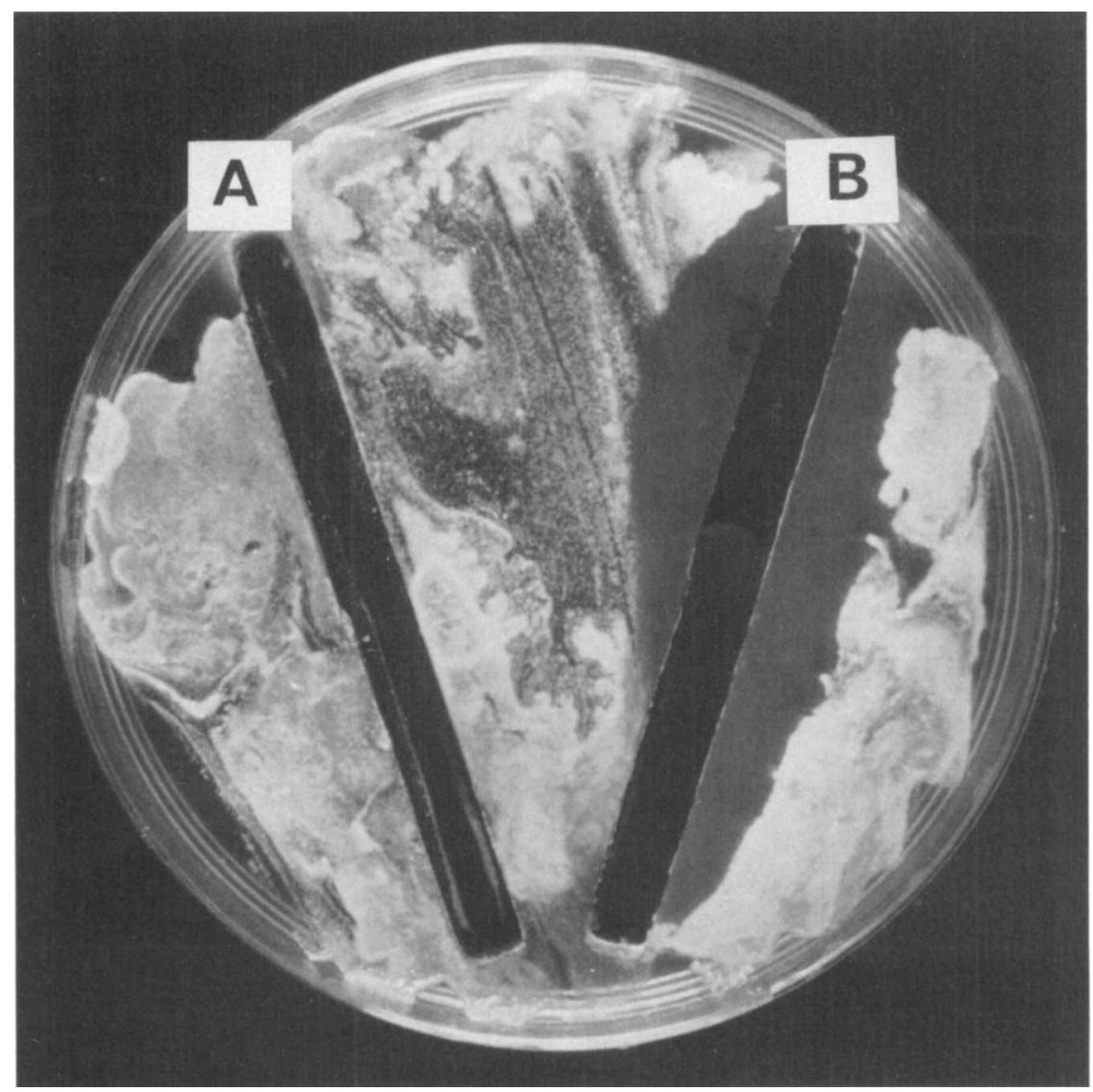

Fig. 2.-Surface growth of $M$. tuberculosis showing protection provided by diffusion of catalase into the medium. Ditch A contained catalase, ditch B contained THB culture of streptococci. 
tuberculosis strain I2646, $21(81 \%)$ to strain B1453 and $22(85 \%)$ to $M$. intracellulare. In contrast, the 26 strains of streptococci failed to inhibit any of the nine strains of mycobacteria by the deferred antagonism test.

There was no significant difference between the activity of complete THB culture of streptococci and washed cells against $M$. intracellulare; however, washed cells were significantly less active against $M$. kansasi $(\mathrm{p}<0.01)$ and strains $12646(\mathrm{p}<0.01)$ and B1453 ( $<<0.05)$ (table V). Tests on culture filtrates failed to detect antagonism. The effect of catalase on antagonism by streptococci against catalase-positive (I2646) and catalase-negative (B1453) strains and the highly susceptible $M$. intracellulare are presented in table VI. The addition of catalase 100 units $/ \mathrm{ml}$ significantly reduced the number of streptococci inhibiting the catalase-positive strain I2646, but a concentration of catalase 1000 units $/ \mathrm{ml}$ was necessary significantly to reduce inhibition of the highly susceptible strains B1453 and $M$. intracellulare.

Tests in which cultures of mycobacteria were exposed to streptococcal inhibition and diffusion of catalase resulted in neutralisation of antagonism at the point where maximum contact occurred (fig. 2).

The $p \mathrm{H}$ within zones of inhibition on $7 \mathrm{H} 11$ agar plates was not changed sufficiently to account for failure of growth of mycobacteria. Uninoculated agar was found to have a $p \mathrm{H} 6.6$ and the lowest value recorded within zones was $p \mathrm{H} \mathrm{5.8.} \mathrm{Mycobacteria} \mathrm{have}$ been successfully cultured on agar media at $p \mathrm{H} 5 \cdot 5$ (Stottmeier et al., 1967) and on LJ medium adjusted to $\mathrm{pH} 5 \cdot 1$ (Tripathy et al., 1970).

On BBA medium, incubated aerobically, all 26 strains of streptococci yielded growth of black colonies indicative of peroxide production. In contrast, when the tests were performed in anaerobic conditions the same strains failed to produce peroxide.

Tests for susceptibility to hydrogen peroxide gave results similar to previous experience in this laboratory with control strain $\mathrm{H} 37 \mathrm{Rv}(50 \%$ survivors) and $\mathrm{B} 1453$ ( $<0.1 \%$ survivors). $M$. bovis var BCG $(<1 \%$ survivors) and $M$. intracellulare $(<1 \%$ survivors) were highly susceptible to peroxide in these standard test conditions.

\section{DisCUSSION}

The phenomenon of in-vitro bacterial antagonism has been recognised for several decades (Aly and Shinefield, 1982). In most instances antagonism is caused by bacteriocins which act only on strains of the same or closely related species. However, Tagg et al. (1976) considered the bacteriocins of some gram-positive species to be distinct from those of gram-negative species in that they have a wider species activity. Dajani et al. Tom and Law (1976) isolated bacteriocins (termed viridins) from $S$. sanguis and $S$. mitis which were shown to have a broad spectrum of activity including inhibition of several gram-negative species. There have also been several studies in which in-vitro inhibition by $\alpha$-haemolytic streptococci was shown to be due to production of inhibitory concentrations of hydrogen peroxide. Holmberg and Hallander (1973) and LeBien and Bromel (1975) detected an accumulation of hydrogen peroxide in cultures of $S$. sanguis and $S$. mitis. The simultaneous antagonism technique, used in this investigation, detected in-vitro antagonism by streptococci against several species of mycobacteria. However, tests by the deferred antagonism technique failed to detect bacterial-interference-indicating factors other than bacteriocins as the cause of antagonism. It is also unlikely that alteration of the $p \mathrm{H}$ was the cause of streptococcal antagonism. 
The evidence presented here showing that streptococcal inhibition of mycobacterial growth can be prevented or reduced by increasing the concentration of catalase in the medium suggests that peroxide is the most likely cause of antagonism. This conclusion is supported by the finding that the most susceptible strain of $M$. tuberculosis (B1453) is catalase negative and highly sensitive to peroxide. The two other strains found to be highly susceptible to streptococcal antagonism ( $M$. intracellulare and $M$. bovis var BCG) were also highly susceptible to peroxide.

In strains of $M$. tuberculosis, isoniazid resistance accompanied by loss of catalase activity and increased susceptibility to peroxide is well documented (Middlebrook 1954; Knox et al., 1956). Increased susceptibility to peroxide also occurs in isoniazid-sensitive, catalase-positive, strains isolated in Southern India (Mitchison et al., 1963). However, there is scant information regarding the peroxide susceptibility of other species of mycobacteria. It is interesting that $M$. kansasi, the most active catalase producer of the strains tested, was sensitive to $9(35 \%)$ of the streptococci. This observation supports the findings of Jackett et al. (1978) that possession of catalase is only one feature that confers resistance to peroxide. The other factor(s) have not yet been identified.

The finding that washed bacterial cells were less active than complete culture against three of the four strains of mycobacteria tested and that cell-free filtrates were inactive, agrees with the findings of Holmberg and Hallander (1973) who detected peroxide activity in actively growing cultures, but not in bacteria-free filtrates.

It is clear from the results of this investigation that strains with a high degree of susceptibility to peroxide were the most sensitive to streptococcal antagonism. There was, however, no association between antagonism and the degree of peroxide susceptibility among less sensitive strains.

It is of interest that the strains of streptococci examined by Darling and Hart (1976) failed to inhibit growth of $M$. avium and $M$. intracellulare but the strain used by Gelbert et al. (1974) was inhibitory to both species. In the present investigation all 26 strains of streptococci inhibited $M$. intracellulare but not $M$. avium. Because more than 20 serotypes of the $M$. avium-intracellulare complex have been identified (Schaefer, 1979) it would be interesting to examine the susceptibility of a more representative sample. However, peroxide production by streptococci is extremely variable (Thomas and Pera, 1983) and it is unlikely that bacterial interference would provide a sufficiently reproducible method for use as an aid to identification. It would therefore be necessary to develop an in-vitro peroxide-susceptibility test similar to that used by Subbaiah et al. (1960) to detect differences between serotypes of the $M$. avium-intracellulare complex.

Streptococcal contamination of LJ cultures may lead to inhibition of mycobacterial growth either by changes in toxicity of malachite green (Marshall, 1958) or by an accumulation of peroxide. Marks and Thomas (1958) demonstrated the benefits of adding benzylpenicillin to $\mathrm{LJ}$ medium to reduce contamination, and the present investigation supports this recommendation. It is concluded that in-vitro inhibition of mycobacterial growth by streptococci is due to a peroxide-mediated antagonism which serves no useful purpose in the identification or typing of mycobacteria.

\section{REFERENCES}

Allen B W, Swaffield J E 1982 Mycobacterium tuberculosis: recovery from contaminated culture media and identification of bacteria responsible for contamination. Medical Laboratory Sciences 39:11-13. 
Aly R, Shinefield H R 1982 Bacterial interference. C.R.C. Press Inc, Boca Raton, FA, USA.

Dajani A S, Tom M C, Law D J 1976 Viridins, bacteriocins of alpha-hemolytic streptococci: isolation, characterization, and partial purification. Antimicrobial Agents and Chemotherapy 9:81-88.

Darling C L, Hart G D 1976 Antagonistic action of Streptococcus salivarius and Streptococcus faecalis to Mycobacterium tuberculosis. Journal of Clinical Microbiology 4:375-378.

Gelbert S M, Tonaki H, Adams R 1974 An $\alpha$-hemolytic Streptococcus with lytic activity specific for group III mycobacteria. American Review of Respiratory Diseases 109:151-154.

Holmberg K, Hallander H O 1973 Production of bactericidal concentrations of hydrogen peroxide by Streptococcus sanguis. Archives of Oral Biology 18:423-434.

Jackett P S, Aber V R, Lowrie D B 1978 Virulence and resistance to superoxide, low $p \mathrm{H}$ and hydrogen peroxide among strains of Mycobacterium tuberculosis. Journal of General Microbiology 104:37-45.

Knox R, Meadow P M, Worssam A R H 1956 The relationship between the catalase activity, hydrogen peroxide sensitivity, and isoniazid resistance of mycobacteria. American Review of Tuberculosis 73:726-734.

Kraus F W, Nickerson J F, Perry W I, Walker A P 1957 Peroxide and peroxidogenic bacteria in human saliva. Journal of Bacteriology 73:727-735.

LeBien T W, Bromel M C 1975 Antibacterial properties of a peroxidogenic strain of Streptococcus mitior (mitis). Canadian Journal of Microbiology 21:101-103.

Marks J, Thomas C H H 1958 Notes on the cultivation of tubercle bacilli. Monthly Bulletin of the Ministry of Health and the Public Health Laboratory Service 17:194-202.

Marshall J 1958 The effect of contamination on Löwenstein-Jensen medium. Monthly Bulletin of the Ministry of Health and the Public Health Laboratory Service 17:202-206.

Middlebrook G 1954 Isoniazid resistance and catalase activity of tubercle bacilli. American Review of Tuberculosis 69:471-472.

Mitchison D A, Selkon J B, Lloyd J 1963 Virulence in the guinea-pig, susceptibility to hydrogen peroxide, and catalase activity of isoniazid-sensitive tubercle bacilli from South Indian and British patients. Journal of Pathology and Bacteriology 86:377-386.

Schaefer W B 1979 Serological identification of atypical mycobacteria. In: Bergan T, Norris J R (eds) Methods in microbiology, Vol 13. Academic Press, London. pp 323-343.

Stottmeier K D, Beam R E, Kubica G P 1967 Determination of drug susceptibility of mycobacteria to pyrazinamide in 7H10 agar. American Review of Respiratory Diseases 96:1072-1075.

Subbaiah T V, Mitchison D A, Selkon J B 1960 The susceptibility to hydrogen peroxide of Indian and British isoniazid-sensitive and isoniazid-resistant tubercle bacilli. Tubercle 41:323-333.

Tagg J R, Dajani A S, Wannamaker L W 1976 Bacteriocins of gram-positive bacteria. Bacteriological Reviews 40:722-756.

Thomas E L, Pera K A 1983 Oxygen metabolism of Streptococcus mutans: uptake of oxygen and release of superoxide and hydrogen peroxide. Journal of Bacteriology 154:1236-1244.

Tripathy S P, Mitchison D A, Nair N G K, Radhakrishna S, Subbammal S 1970 A comparison of various measures of sensitivity of $M$. tuberculosis to pyrazinamide. Tubercle 51:375-388. 